\title{
Formation and detectability of Earth-like planets around Alpha-Centauri $\mathrm{B}^{\dagger}$
}

\author{
Erica Davis ${ }^{1}$ \\ ${ }^{1}$ Department of Astronomy and Astrophysics, University of California, Santa Cruz \\ 1156 High Street, Santa Cruz, California 95064 \\ email: edavis@ucolick.org
}

\begin{abstract}
We simulate the late stages of planet formation around Alpha Centauri B and analyze the detectability of the resulting terrestrial planet systems. The N-body accretionary evolution of a $\Sigma \propto r^{-1}$ disk populated with 400-900 lunar-mass oligarchs is followed for $200 \mathrm{Myr}$ for each simulation. All of eight runs result in the formation of multiple-planet systems with at least one planet in the 1-2 $\mathrm{M}_{\oplus}$ mass range at $0.5-1.5 \mathrm{AU}$. We examine the detectability of our simulated planetary systems by generating synthetic radial velocity observations including noise based on the radial velocity residuals to the recently published three planet fit to the nearby K0V star HD 69830. Using these synthetic observations, we find that we can reliably detect a $1.8 \mathrm{M}_{\oplus}$ planet in the habitable zone of $\alpha$ Centauri B after only three years of high cadence observations. We also find that the planet is detectable even if the radial velocity precision is $3 \mathrm{~m} \mathrm{~s}^{-1}$, as long as the noise spectrum is white.
\end{abstract}

Keywords. binaries: general, planetary systems: formation, stars: individual ( $\alpha$ Centauri B)

\section{Introduction}

In the past decade, over 250 extrasolar planets have been discovered in a plethora of diverse environments. Earth-like planets in habitable-zone orbits, however, remain well below the threshold of detection. A good representation of the Doppler velocity stateof-the-art is presented by the triple planet system orbiting HD 69830. This system has been shown to contain three Neptune-mass planets, including one on a 197-day orbit, all revealed after only 74 radial velocity observations with residual noise of $0.6 \mathrm{~ms}^{-1}$ (Lovis et al. 2006). The detection of the HD 69830 system suggests that focused efforts on selected stars may be able to probe down to the characterization of planets with radial velocity half-amplitudes considerably below $1 \mathrm{~m} \mathrm{~s}^{-1}$. The $\alpha$ Centauri system provides a unique opportunity to push the limits of Doppler detection in the search for terrestrial planets in habitable orbits.

Studies of $\alpha$ Cen A and B show that terrestrial planet formation is possible around both stars despite their strong binary interaction (Quintana et al. 2002, 2006, 2007). Results to date indicate that planetary systems with one or more Earth-mass planets can form within 2.5 AU from the host stars and remain stable for gigayear scales (Quintana et al. 2007). Numerical simulations and stability analyses of planetesimal disks indicate that material is stable within $3 \mathrm{AU}$ of $\alpha$ Cen $\mathrm{B}$ as long as the inclination of the disk with respect to the binary is $\leqslant 60^{\circ}$ (Quintana et al. 2002; Wiegert \& Holman 1997).

We assess the detectability of terrestrial planets around $\alpha$ Cen B. Eight simulations of the late stage of planet formation are carried out, and each resulting planetary system is tested for detectability using a Monte Carlo method for generating synthetic radial

$\dagger$ More complete results have been submitted for publication: Guedes et al. 2007, ApJ. 


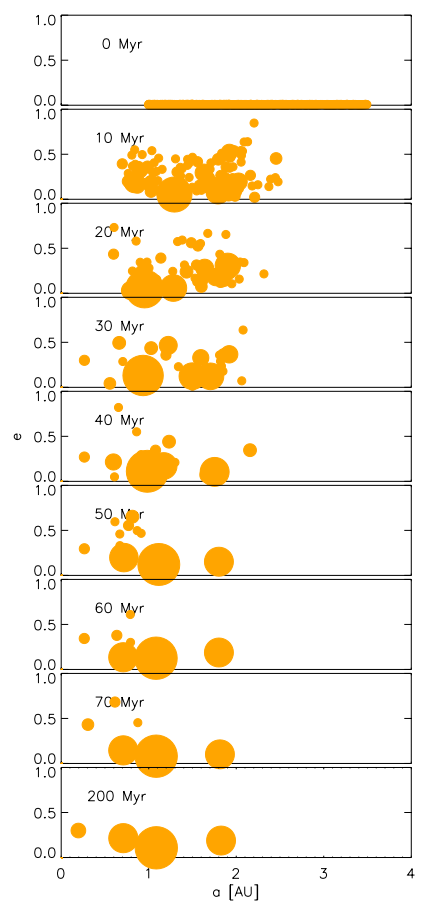

Figure 1. Evolution of a circumstellar disk initially populated by moon-mass oligarchs in circular orbits around $\alpha$ Centauri B. The radius of each circle is proportional to the size of the object.

velocity observations. Based on our results, we are able to accurately evaluate the detectability of planetary systems around the star.

\section{Terrestrial Planet Formation}

The initial conditions of the circumstellar disk in our simulations mimic conditions at the onset of the chaotic growth phase of terrestrial planet formation (Kokubo \& Iba 1998, Kenyon \& Bromley 2006) in which collisions of isolated oligarchs, protoplanets of approximately lunar mass, dominate the evolution of the disk. At the start of this phase, several hundred oligarchs orbit the star on nearly circular orbits. Gravitational interactions among oligarchs work to form the final planetary system around the star and clear out remaining material from the disk.

We model the $\alpha$ Centauri B circumstellar disk with a $\Sigma=\Sigma_{0}(a / 1 A U)^{-1}$ surface density profile where $\Sigma_{0}=8.4-18.8 \mathrm{~g} \mathrm{~cm}^{-2}$ as calculated from the total mass $M=N m$, where $N$ is the number of oligarchs in the disk and $m$ is the oligarch's mass. The disk extends from $1<a<3.5 \mathrm{AU}$ and it is coplanar with the binary orbit. For each run, we populate the disk with $N=400$ to $N=900$ oligarchs of lunar mass $\left(0.0123 M_{\oplus}\right)$ with semi-major axes chosen via a rejection method in $a$ to obtain a $\Sigma \propto r^{-1}$ density profile.

Initial orbital elements of each oligarch are randomly generated with mean anomalies, arguments of pericenter, and longitudes of ascending node extending from $0^{\circ}$ to $360^{\circ}$, eccentricities in the range $0<e<0.001$, and inclinations in the range $0^{\circ}<i<1^{\circ}$ with respect to the plane of the binary. 
Each integration was run for $200 \mathrm{Myr}$ using a specialized version of the symplectic hybrid integrator in the MERCURY integration package (Chambers 1999). This $N$ body code is designed to study planet growth in the presence of a binary companion (Chambers et al. 2002). Bodies grow via accretion through perfectly inelastic embryoembryo collisions, and, therefore, close encounters are integrated directly rather than symplecticaly.

Figure 1 shows the late evolutionary stage of a protoplanetary disk initially containing 600 moon-mass embryos (run03). Bodies in the outer parts of the disk $(a>3 \mathrm{AU})$ are immediately launched into highly eccentric orbits and either migrate inward to be accreted by inner bodies, collide with the central star, or are ejected from the system $\left(a_{e j}=100 \mathrm{AU}\right)$. In this simulation, $\sim 65 \%$ of the total initial mass is cleared within the first 70 Myr. By the end of simulation run03, four planets have formed. One planet has approximately the mass of Mercury and is located at $a=0.2 \mathrm{AU}$, two $0.6 M_{\oplus}$ planets form at $a=0.7$ and $a=1.8 \mathrm{AU}$, and a $1.8 M_{\oplus}$ planet forms at $a=1.09 \mathrm{AU}$.

Table 1 shows the orbital elements of the final systems that emerge from the calculations. All of our simulations result in the formation of 1-4 planets with semi-major axes in the range $0.7<a<1.9 \mathrm{AU}$, in agreement with Quintana et al. (2002). We find that $42 \%$ of all planets formed with masses in the range $1-2 M_{\oplus}$ reside in the star's habitable zone (Fig. 2), which is taken to be $0.5 \mathrm{AU}<a_{h a b}<0.9 \mathrm{AU}$ (Kasting et al. 1993).

\section{Detectability}

We took the orbital elements of the systems emerging from our simulations and generated model radial velocities. We developed a code which effectively simulates observing conditions for any specific location on Earth. Given the latitude and longitude of an observatory and the RA and DEC of an object, the code determines when an object is observable. Two additional inputs concern the beginning and end of an observing night: the angle of the sun below the local horizon and the maximum airmass of the object, beyond which observing should not continue. We assume $25 \%$ of the observing nights are lost. This accounts for adverse weather conditions and other effects which could result in missed observations. We assumed access to a dedicated telescope at Las Campanas Observatory. At this location, $\alpha$ Centauri is observable for about 10 months out of the

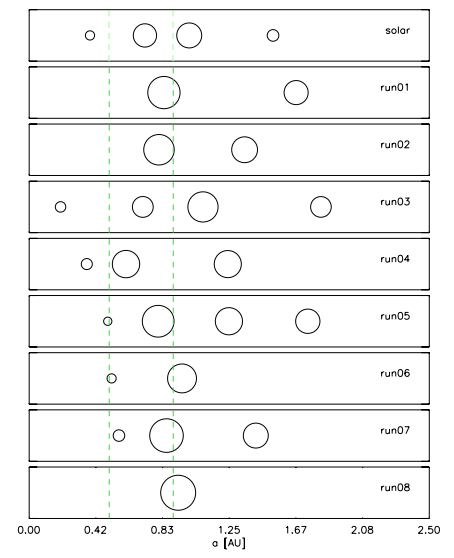

Figure 2. Results from runs 1 through 8 . Each panel shows the resulting planetary system and the habitable zone (green dashed lines) for the K1 star $\alpha$ Cen B. 
Table 1. Simulation Results

\begin{tabular}{|c|c|c|c|c|c|c|c|}
\hline Run & $N_{0}^{a}$ & planet & $M\left[M_{\oplus}\right]$ & Period $[y \mathbf{y}]$ & $a[\mathbf{A U}]$ & $e$ & $I\left[^{\circ}\right]$ \\
\hline 01 & 700 & $\begin{array}{l}\mathrm{a} \\
\mathrm{b}\end{array}$ & $\begin{array}{l}0.897 \\
2.165\end{array}$ & $\begin{array}{l}2.262 \\
0.812\end{array}$ & $\begin{array}{l}1.669 \\
0.843\end{array}$ & \begin{tabular}{|l|}
0.198 \\
0.142
\end{tabular} & $\begin{array}{l}4.965 \\
4.516\end{array}$ \\
\hline 02 & 700 & $\begin{array}{l}\mathrm{a} \\
\mathrm{b}\end{array}$ & $\begin{array}{l}1.820 \\
1.107\end{array}$ & $\begin{array}{l}0.767 \\
1.640\end{array}$ & $\begin{array}{l}0.811 \\
1.346\end{array}$ & $\begin{array}{l}0.016 \\
0.032\end{array}$ & $\begin{array}{l}1.846 \\
3.064\end{array}$ \\
\hline 03 & 600 & $\begin{array}{l}a \\
b \\
c \\
d\end{array}$ & $\begin{array}{l}0.565 \\
0.578 \\
0.073 \\
1.771\end{array}$ & $\begin{array}{l}2.585 \\
0.628 \\
0.091 \\
1.189\end{array}$ & $\begin{array}{l}1.831 \\
0.710 \\
0.196 \\
1.086\end{array}$ & \begin{tabular}{|l}
0.181 \\
0.242 \\
0.286 \\
0.031
\end{tabular} & $\begin{array}{l}3.979 \\
6.827 \\
7.590 \\
3.124\end{array}$ \\
\hline 04 & 800 & $\begin{array}{l}\mathrm{a} \\
\mathrm{b} \\
\mathrm{c}\end{array}$ & $\begin{array}{l}0.086 \\
1.316 \\
1.279\end{array}$ & $\begin{array}{l}0.227 \\
0.495 \\
1.453\end{array}$ & $\begin{array}{l}0.361 \\
0.606 \\
1.242\end{array}$ & $\begin{array}{l}0.244 \\
0.105 \\
0.168\end{array}$ & \begin{tabular}{|c}
19.135 \\
1.639 \\
2.042
\end{tabular} \\
\hline 05 & 900 & $\begin{array}{l}a \\
b \\
c \\
d\end{array}$ & $\begin{array}{l}2.054 \\
0.922 \\
0.036 \\
1.291\end{array}$ & $\begin{array}{l}0.760 \\
2.412 \\
0.361 \\
1.464\end{array}$ & $\begin{array}{l}0.806 \\
1.734 \\
0.491 \\
1.248\end{array}$ & $\begin{array}{l}0.052 \\
0.051 \\
0.094 \\
0.145\end{array}$ & \begin{tabular}{|c}
1.585 \\
5.784 \\
18.108 \\
5.391
\end{tabular} \\
\hline 06 & 400 & $\begin{array}{l}\mathrm{a} \\
\mathrm{b}\end{array}$ & $\begin{array}{l}1.549 \\
0.049\end{array}$ & $\begin{array}{l}0.981 \\
0.388\end{array}$ & $\begin{array}{l}0.956 \\
0.515\end{array}$ & $\mid \begin{array}{l}0.095 \\
0.345\end{array}$ & \begin{tabular}{|c|}
4.777 \\
15.378
\end{tabular} \\
\hline 07 & 800 & $\begin{array}{l}\mathrm{a} \\
\mathrm{b} \\
\mathrm{c}\end{array}$ & $\begin{array}{l}0.996 \\
0.098 \\
2.435\end{array}$ & $\begin{array}{l}1.769 \\
0.441 \\
0.835\end{array}$ & $\begin{array}{l}1.419 \\
0.663 \\
0.858\end{array}$ & $\begin{array}{l}0.169 \\
0.325 \\
0.024\end{array}$ & \begin{tabular}{|l}
6.034 \\
8.259 \\
3.759
\end{tabular} \\
\hline 08 & 700 & $\mathrm{a}$ & 2.755 & 0.944 & 0.931 & 0.217 & 4.391 \\
\hline
\end{tabular}

Notes:

${ }^{a}$ Initial number of oligarchs.

year. We assumed an observing cadence of one exposure every 200 seconds, corresponding to the read out time of the detector (this is a conservative estimate, since in practice we would expect a considerably higher duty cycle). Finally, we added various values of Gaussian white noise to the model radial velocities.

Figure 3 shows a naïve result for the observed synthetic radial velocities for the system that arose from run03. In this case, we assumed Gaussian white noise with amplitude

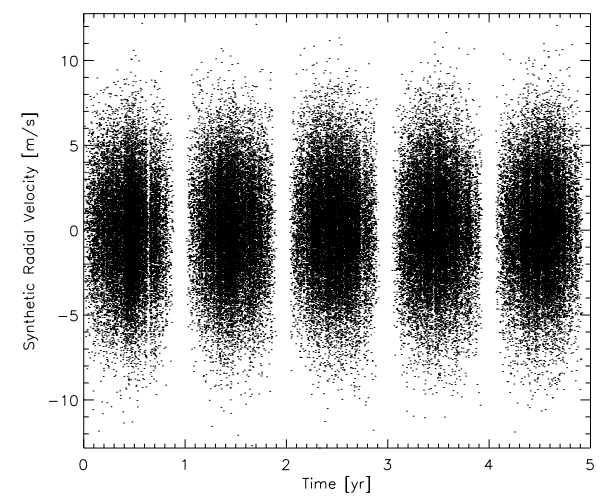

Figure 3. Synthetic radial velocities over a five year period. The 60-day gaps in the data account for the time period when the system is below the horizon. Other gaps in the data emulate nights of missed observations due to bad weather and other adverse events. 


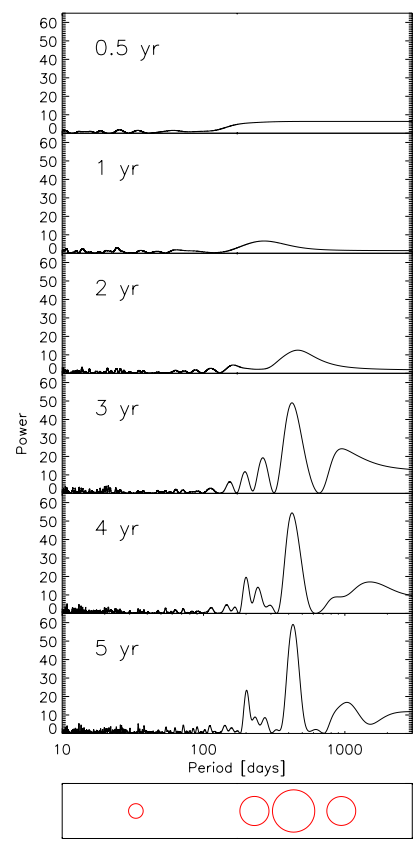

Figure 4. Evolution of the periodogram for run03 over 5 years as 97,260 synthetic radial velocity observations are made, assuming Gaussian white noise with amplitude $3 \mathrm{~m} \mathrm{~s}^{-1}$. The $1.7 M_{\oplus}$ planet $(\mathrm{P}=1.2 \mathrm{yr})$ could be confidently detected in 3 years.

$3 \mathrm{~ms}^{-1}$. In keeping with the fact that the star is a virtual twin of HD 69830 (Lovis et al. 2006), $\alpha$ Cen B is expected to show very little radial velocity noise or jitter. The detection of three Neptune mass planets around HD 69830 was facilitated by the small instrumental uncertainties for its radial velocity observations, with a median value of $\approx 0.7 \mathrm{~m} \mathrm{~s}^{-1}$, along with the assumption of a very small stellar jitter. It is important to note that the model radial velocity for $\alpha$ Cen B due to the four terrestrial planets in this system has an amplitude of $23 \mathrm{~cm} \mathrm{~s}^{-1}$, or a factor of 13 below the noise.

Over the five year span, 97,260 measurements can be obtained. For this example, we find that we can confidently detect 2 or 3 planets in this time span. We can confidently detect at least one of the planets after the first three years of observations. Figure 4 shows the periodogram of the synthetic radial velocities after $0.5,1,2,3,4$, and 5 years of observations for this system with $3 \mathrm{~m} \mathrm{~s}^{-1}$ white noise. These findings, along with the results from our eight terrestrial planet formation simulations and those of Quintana et al. (2007) suggest that we may detect a habitable terrestrial planet around one of our nearest stellar neighbors in less than 3 years of observation.

\section{Discussion}

The possibility that detectable terrestrial planets are orbiting $\alpha$ Cen B does much to fire the imagination, and, indeed, a positive identification of such a planet would be a truly landmark discovery. $\alpha$ Cen's proximity allows one to envision space-based 
follow-up efforts (astrometric, coronographic, interferometric) to characterize the planets that would be far more difficult to carry out for planets orbiting less luminous and more distant stars.

To phrase the situation another way, our current understanding of the process of terrestrial planet formation suggests that both components of the $\alpha$ Cen system may have terrestrial planets, provided that disks formed around the stars and that the system has not undergone strong interactions with other stars over the lifetime of the binary. Furthermore, assuming a negligible degree of red noise on the ultra-low frequency range for the host star, successful detection of such terrestrial planets orbiting $\alpha$ Cen B can be made within a few years and with the modest investment of resources required to mount a dedicated radial-velocity campaign with a 1-meter class telescope and high-resolution spectrograph. A lack of planets orbiting these stars would, thus, either provide insight into the history and physical parameters of the $\alpha$ Cen system or provide a critical hint that there is a significant qualitative gap in our understanding of terrestrial planet formation.

\section{References}

Chambers, J. E. 1999, MNRAS, 304, 793

Chambers, J. E., Quintana, E. V., Duncan, M. J., \& Lissauer, J. J. 2002, AJ, 123, 2884

Kasting, J. F., Whitmire, D. P., \& Reynolds, R. T. 1993, Icarus, 101, 108

Kenyon, S. J. \& Bromley, B. C. 2006, AJ, 131, 1837

Kokubo, E. \& Ida, S. 1998, Icarus, 131, 171

Lovis, C. et al., 2006, Nature, 441, 305

Quintana, E. V., Lissauer, J. J., Chambers, J. E., \& Duncan, M. J. 2002, ApJ, 576, 982

Quintana, E. V. \& Lissauer, J. J. 2006, Icarus, 185, 1

Quintana, E. V., Adams, F. C., Lissauer, J. J., \& Chambers, J. E. 2007, ApJ, 660, 807

Wiegert, P. A. \& Holman, M. J. 1997, AJ, 113, 1445 